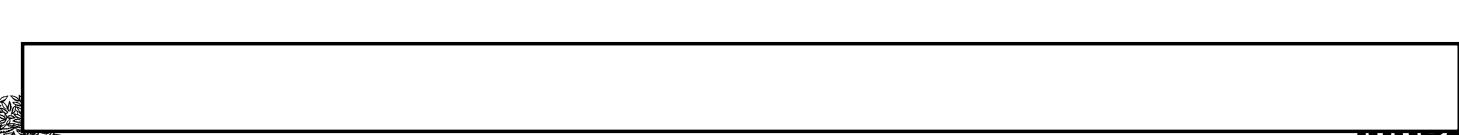

\title{
A semi-in-vivo preparation for optical recording of the insect brain
}

\author{
C. Giovanni Galizia *, Jasdan Joerges, Armin Küttner, Till Faber, Randolf Menzel \\ Institut für Neurobiologie, Freie Universität Berlin, Königin Luise Str. 28-30, 14195 Berlin, Germany
}

Received 25 February 1997; accepted 13 April 1997

Konstanzer Online-Publikations-System (KOPS)

URL: http://www.ub.uni-konstanz.de/kops/volltexte/2007/2267/

URN: http://nbn-resolving.de/urn:nbn:de:bsz:352-opus-22671

\begin{abstract}
We describe a method for optically recording neuronal activity from an intact insect brain, upon natural sensory stimulation. In this preparation, the head capsule of the honeybee, Apis mellifera, is isolated from the body while leaving the entire brain undamaged. In short, a hole is first cut into the cuticule to allow optical access to the brain and to allow the removal of tracheae and glands. Then the head is cut free and placed into a dye-loaded and cooled ringer solution in a staining chamber for $1 \mathrm{~h}$. Subsequently, the head is fixed in a recording chamber, covered with a cover-slip, and imaged under the microscope with a cooled CCD camera. The whole preparation leaves the antennae dry, free to move, and functional throughout the experiment, allowing for natural odour stimulation of the olfactory system. Using calcium sensitive or potential sensitive dyes (calcium-green or RH795), we could record the processing of olfactory information at the glomerular level in the antennal lobe of the bee. (C) 1997 Elsevier Science B.V.
\end{abstract}

Keywords: Optical imaging; Calcium green; RH795; Olfaction; Antennal lobe; Apis mellifera

\section{Introduction}

The olfactory system represents a neuronal multidimensional parallel processing system par excellance. Information is gathered via a large number of different receptor cell types, and processed in primary neuropils that are glomerular structures (i.e., the antennal lobe in insects, which is the functional analogue to the olfactory bulb in mammals) (Boeckh et al., 1990). It is assumed that olfactory information is coded in across fiber activity patterns of the projection neurones that leave these primary processing centres (Shepherd, 1993). How this coding process is achieved is yet unclear. However, the investigation into these mechanisms will enormously benefit from methods that allow the simultaneous measuring of neural activity across wide portions of the brain areas involved.

Optical recording of neuronal activity in intact nervous tissues is an increasingly important and widely used tool to study the brain, both in vertebrates (Bon-

\footnotetext{
* Corresponding author.
}

hoeffer et al., 1995; Ts'o et al., 1990; Lieke et al., 1989; Blasdel, 1992) and invertebrates (Joerges et al., 1997; Cohen, 1989; Joerges et al., 1995; Kleinfeld et al., 1994). It allows the simultaneous measurement of the summated activity of entire neuropils, with good spatial and reasonable temporal resolution. Neuronal signals can be registered optically by exploiting either intrinsic changes in tissue properties (Grinvald et al., 1986; Bonhoeffer and Grinvald, 1993), using voltage sensitive dyes (Blasdel, 1992) or calcium sensitive dyes (Yuste et al., 1995; Yuste and Katz, 1991; Borst and Egelhaaf, 1994). As compared to measuring single cell activities, monitoring an entire tissue is accompanied with a drop in both signal amplitude and signal to noise ratio, and with an increase in disturbing side effects: movement artifacts are of particular concern to in vivo optical physiologists. Furthermore, access to the brain can be geometrically difficult: only the superficial layers of the brain are normally accessible, and staining may be spoiled by the excess tissue of the entire body.

In vitro preparations have been successfully used in optical physiology: slice preparations preserve a good 

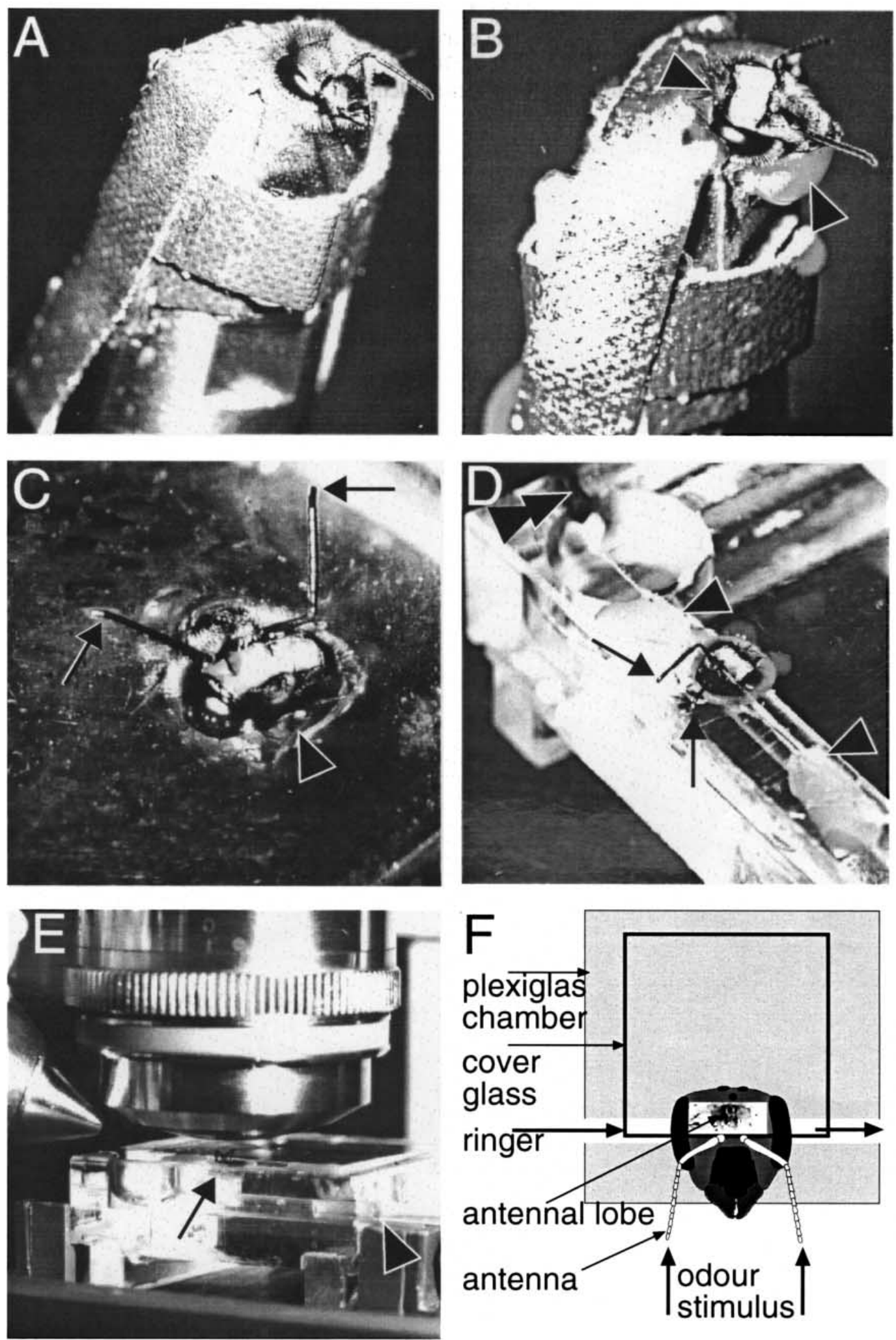

Fig. 1.

deal of intrinsic structure, and allow for good staining and optical imaging (Yuste et al., 1995; Yuste and Katz, 1991). In insects, brain preparations have been used to study the physiology of single cells
(Brotz et al., 1995). The goal of such studies is to exploit the advantages of slice preparations, while preserving as much as possible the intrinsic connectivity of the brain. 
We have developed a semi-in-vivo preparation of the honeybee (Apis mellifera) brain that leaves the entire brain and olfactory system intact, including the receptor cells on the antenna, the antennae themselves, the antennal lobes and the mushroom bodies. This preparation allows the functional imaging of the brain while at the same time naturally stimulating the receptor cells with odours. Using this preparation and staining with either calcium sensitive dyes or potential sensitive dyes we were able to measure the olfactory code of the honeybee in the antennal lobes.

\section{Materials and methods}

In this methodological paper, we will emphasize particularly the preparation and staining protocol. Recording and data processing methods correspond to standard optical recording methodology, and are reported in succinct form.

\subsection{The preparation}

Foraging bees were collected shortly before the experiment from the bee hive. After cooling of the bee to between 4 and $6^{\circ} \mathrm{C}$, it was fixed in a metal tube using adhesive tape (See Fig. 1A, photograph of a bee in a metal tube). Then, the head was fixed with wax, taking care not to overheat the bee head. Using a broken razor blade, a small opening was cut in the cuticle covering the head ranging from behind the antenna to the central ocellus on the dorso-ventral axis, and one complex eye to the other on the sides (Fig. 1B). Tissue covering the brain was then removed, including glands, tracheae, and the neurolemma. Great care was taken not to damage the brain. Now, using a razor blade, the bee was decapitated starting between the proboscis and the antennae, and proceeding posteriorly, thus isolating the entire head capsule from the rest of the body. At this stage, the brain is intact in the isolated head capsule with the freely moving antennae attached to it, while the proboscis has been removed, and muscles leading to it have been cut. The brain is then washed in fresh ringer solution $(130 \mathrm{mM} \mathrm{NaCl}, 6 \mathrm{mM} \mathrm{KCl}, 4 \mathrm{mM}$ $\mathrm{MgCl}_{2}, 5 \mathrm{mM} \mathrm{CaCl}_{2}, 160 \mathrm{mM}$ sucrose, $25 \mathrm{mM}$ glucose, $10 \mathrm{mM}$ HEPES, pH 6.7, $500 \mathrm{mOsmol}$, all chemicals from Sigma, Deisenhofen, Germany) in order to remove proteases and other enzymes that may have been released by glands or the cut oesophagus.

\subsection{The staining process}

The isolated head is then placed in the staining chamber. The camber consists of a dip cut in Sylgard (Dow Corning, Seneffe, Belgium), containing $200 \mu 1$ staining liquid (See Fig. 1C). The bee head is left floating on the staining solution, taking great care to keep the antennae dry. The staining chamber with the bee head is kept at a temperature between 6 and $8^{\circ} \mathrm{C}$ for about $1 \mathrm{~h}$ in a dark box.

Calcium sensitive dyes (calcium-green-1-AM or calcium-green-2-AM, Molecular Probes, Eugene) were first dissolved in 20\% Pluronic F-127 in DMSO (Molecular Probes, Eugene) (50 $\mu \mathrm{g}$ dye in $50 \mu 1$ liquid), and then diluted adding $950 \mu 1$ ringer solution (to a final concentration of $28 \mu \mathrm{M}$; see above for ringer composition). Voltage sensitive dyes (RH795, Molecular Probes, Eugene) were first dissolved in 70\% ethanol, and then diluted adding ringer solution to reach a final concentration of $20 \mu \mathrm{M}$.

\subsection{The recording chamber}

After staining, the bee head is placed in the recording chamber (Fig. 1D, and schematic view in Fig. 1F). The cuticle is mechanically fixed to the plexiglas chamber using dental wax (Deiberit 502, Bohme, Bad Sachsa, Germany). The opening in the cuticle is closed with a cover glass, in order to avoid surface fluctuations during the imaging process, and the cover glass is waxed to the plexiglas chamber. From now on, the brain is constantly perfused with cooled $\left(18-20^{\circ} \mathrm{C}\right)$ and air

Fig. 1. Illustration of the preparation. (A) Living bee fixed in a metal tube. The tube has two parallel stripes of brass fixed to it at the top which leave a gap where the neck of the bee can be inserted. The head is blocked at the back with a narrow stripe of tape. The metal tube is then fixed and the brain is made free under cold anaestesia. (B) Living bee fixed in a metal tube. The bee is fixed to the brass tube with wax along the ocelli and the mouthpieces (arrowheads). Following this, a window is cut into the cuticule. Glands and tracheae are removed to expose the brain. (C) Staining chamber. After isolating the head capsule with the intact brain using a sharp razor blade, it is translocated to the staining chamber. The chamber has a dip cut into Sylgard. The chamber contains bee ringer solution (arrowhead), loaded with vital dyes, e.g. calcium-green-AM for intracellular calcium measurements, or RH795 for membrane potential measurements. Great care is taken to keep the antenna dry (arrows), and the head floating. (D) Bee-head fixed in the recording chamber. A cover glass is fixed over the brain using wax (arrowheads), and the head capsule itself is constantly perfused in ringer solution. Drainage drops are avoided using a woolen thread (top right in the photograph, double arrowhead). Note the free optical access to the brain through the cover glass. The antennae are freely moving in air (arrows), and not affected by the preparation and the perfusion. (E) Recording chamber under the microscope. Imaging is done using a $40 \times$ objective (top), while stimulating with odours (snout of the olfactometer on the left, compare with Fig. 2). Note the antennae in the middle of the photograph (arrows). Ringer influx tube can be seen on the right (arrowhead). (F) Schematic view of the recording chamber as seen from the top, showing the stimulus delivery (air flow, containing odours when stimulating), ringer perfusion, and position of the brain in the chamber while recording. The width of a bee-head is around $3.7 \mathrm{~mm}$. 
saturated ringer solution (see above for ringer composition). To prevent pulsations from a peristaltic pump, perfusion is gravity driven. The chamber is fixed under the microscope and ready for imaging (Fig. 1E).

Natural stimulation with odours is accomplished using a custom built olfactometer (Fig. 2C). The olfactometer delivers a constant air stream to the antennae between stimuli. With the aid of computer controlled magnetic valves (Lee, Westbrook, Connecticut), the air stream is switched to an odourous one when stimulating, and back to the clean air stream after the $2 \mathrm{~s}$ stimulus (Fig. 2A and 2B). Filter papers $\left(1 \mathrm{~cm}^{2}\right)$ are soaked with the odours $(5 \mu 1$ of pure odourant. Citral, hexanal, hexanol, octanol were purchased from Sigma, carnation, lavender and orange extracts were purchased from a local drug store) and then placed in a syringe (volume $0.5 \mathrm{ml}$ ) in the revolver of the olfactometer. The control air stream syringe contains a clean filter paper. Odour mixtures can be applied by simultaneously opening the air stream through two syringes (data not shown). When no stimulation is applied, the front ends of the odour laden syringes are prevented from leaking odours by an air stream perpendicular to their openings, which is constantly removed from the whole apparatus (Fig. $2 \mathrm{~A}$ and $2 \mathrm{~B})$.

\subsection{Data collection and processing}

We used an upright microscope (Axioskop, Zeiss, Oberkochen, Germany) with a fixed object stage, and a long distance $40 \times$ air objective (Zeiss, LD Acroplan, NA $=0.6$ ). Filter sets for calcium green are BP 450-490 excitation filter, 510 dicroic, BP 515-565 fluorescence filter. Filter sets for RH795 consisted of BP 546 excitation filter, 580 dicroic, LP 590 fluorescence filter. Probes were illuminated using a $100 \mathrm{~W}$ halogen lamp (64458 AX, Philips) with a stabilized power supply unit (68830, LOT Oriel, Darmstadt, Germany). Optical signals are recorded using a cooled slow-scan 12 bit CCD Camera (CH250A, Photometrics, Tucson, Arizona), recording between two and four frames per second. Neighbouring pixels were binned on chip to increase measurement speed, and to reduce noise levels. Final image sizes were usually $50 \times 50$ pixels, for an imaged field of $250 \times 250 \mu \mathrm{m}$. The measurements are controlled from a computer, which coordinates light shutter, camera shutter, stimulus delivery via the olfactometer, and data read-out from the camera. Signals $(\mathrm{dF} / \mathrm{F})$ are calculated from the digitally stored light intensity levels using a selfwritten program (written in IDL, Interactive Display Language, Research Systems, Boulder, Colorado). For voltage sensitive dye recordings, an average of between eight and 16 trials was necessary to reach a

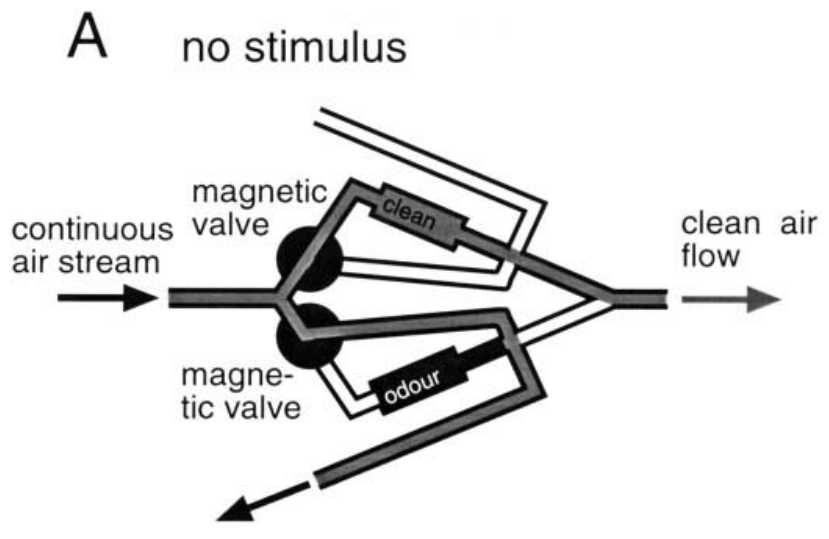

B odour stimulus
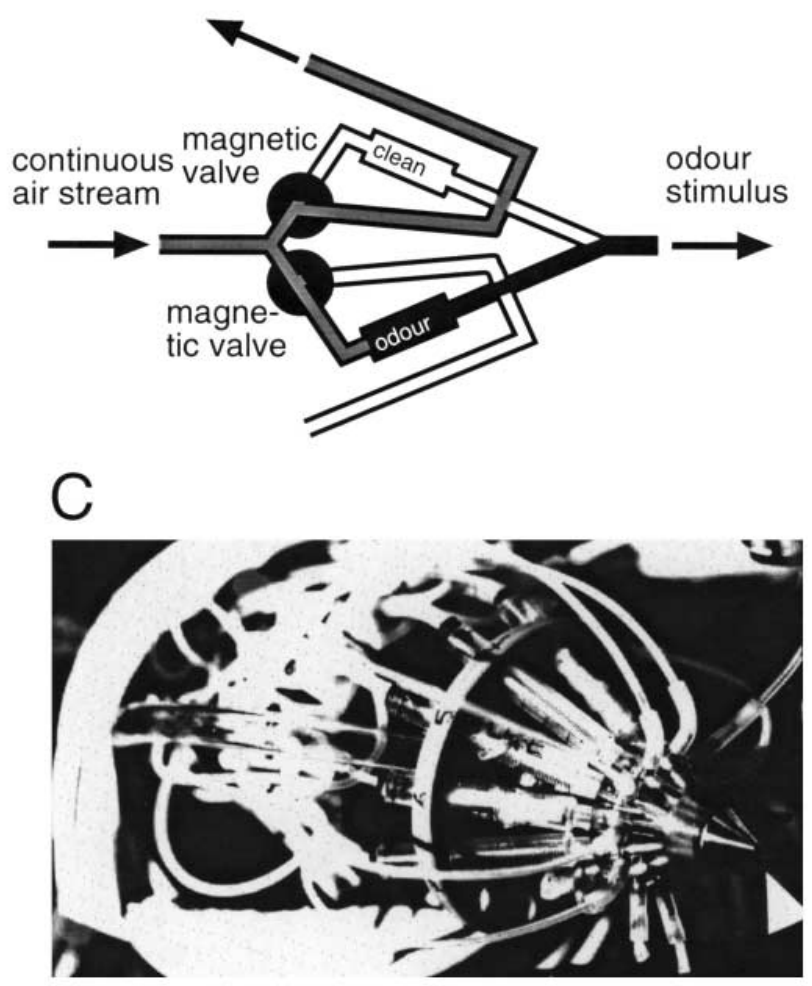

Fig. 2. Schematic of the olfactometer employed. (A) Situation when no stimulation is given. A continuous air stream is delivered, through a clean syringe, to the antennae of the bee (air flow). At the same time, a constant, clean air current passes perpendicularly to the connection of the odour laden syringes (only one shown here) with the apparatus exit, in order to avoid any leakage of odourant substances into the clean air flow. (B) Situation when stimulating. The magnetic valve of the clean air stream is switched, deviating the air stream along the perpendicular route, while the odour laden pathway is open. All other odourant syringes are closed by the perpendicular air stream at the same time. Eight syringes are combined in one revolver: one for background air flow, 1 for control air stimulation, and six for different odour stimuli. (C) Photograph of the olfactometer. A maximum of 12 syringes (one is marked with an arrow) can be fixed on a revolver. The air stream leaves the olfactometer through the snout, to the right (arrowhead). 

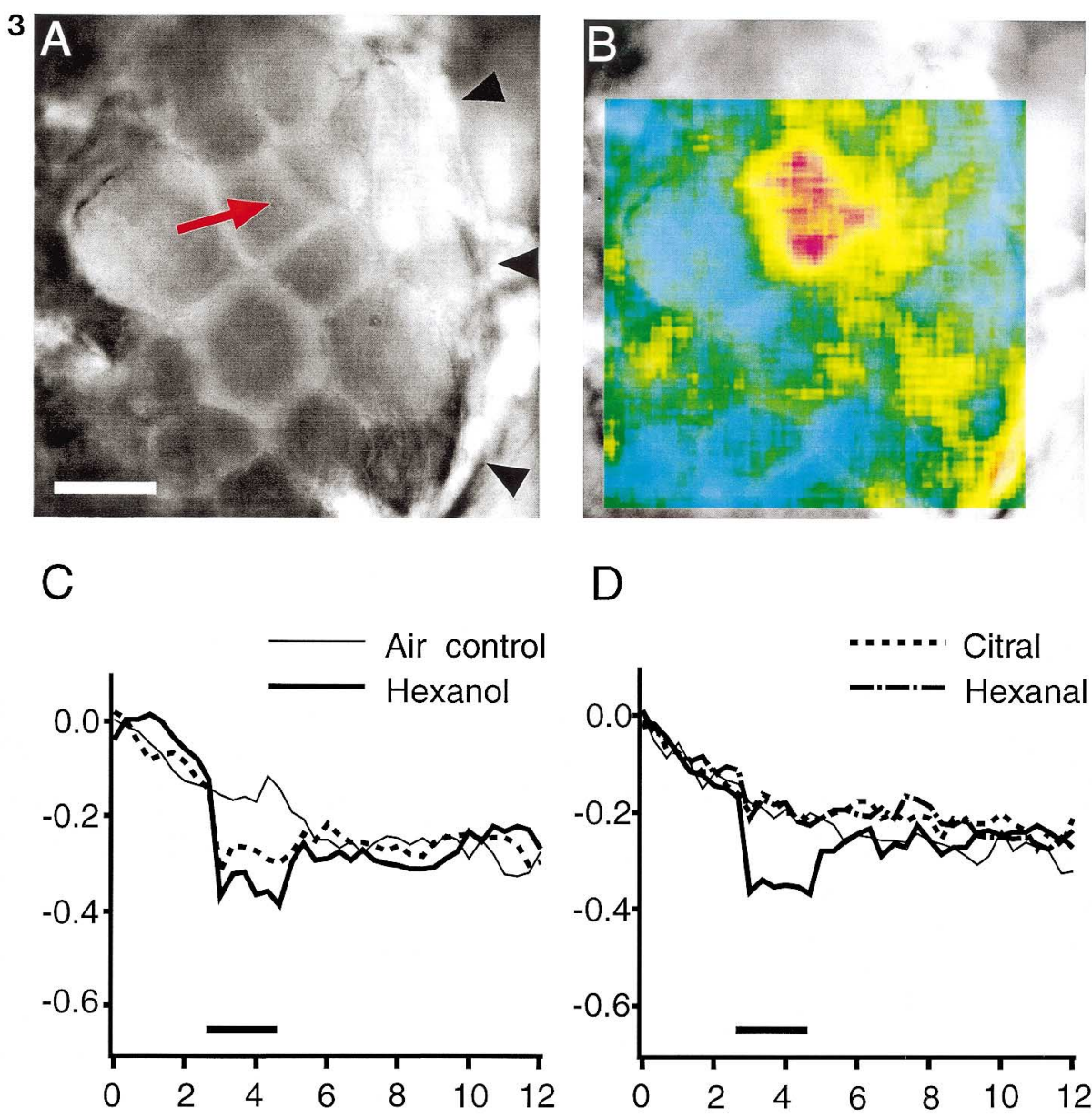

D

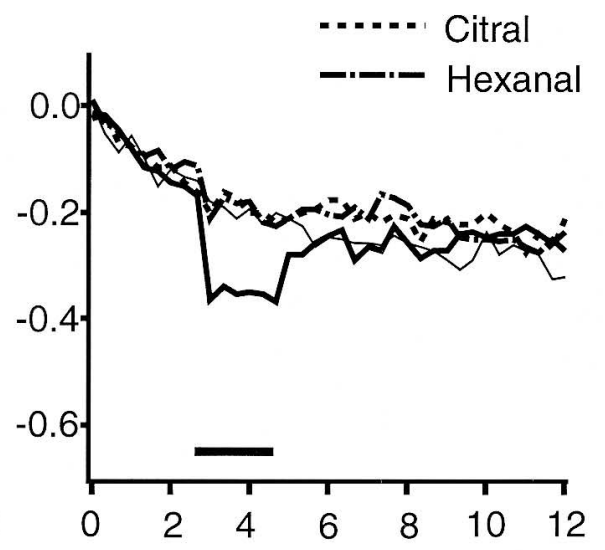

Fig. 3. Voltage sensitive dyes (RH795). (A) Staining with the membrane binding dye RH795 gives clear staining of the glomerular structure of the antennal lobe. This is the view of a left antennal lobe, with a $40 \times$ objective. In all figures, ventral is up, dorsal down, i.e. the antennal nerve points upwards (outside the imaged frame). Scale bar $50 \mu \mathrm{m}$. Note the border of the antennal lobe (arrowheads), and the different shape and size of the various glomeruli. Compare the glomerulus marked with the red arrow with the physiological results in Fig. 3B. (B) Same view as in A, but with a superimposed false-colour coded activity map. Red indicates strong membrane depolarization (reduced flourescent light, due to the shift of the fluorescent emission spectrum of RH795), blue indicates no change in membrane potential (same sequence of colours as in Fig. 4). Only part of the view field has been measured in this experiment. The antennae have been stimulated for $2 \mathrm{~s}$ with hexanal, the false colour coded image gives the mean change in fluorescence during the stimulus interval. Note that the change in activity is strongly focalized to a single glomerulus, marked with a red arrow in A. (C, D). Examples of time-courses of measurements with voltage-sensitive dyes in the antennal lobe. Relative changes in fluorescence $(\mathrm{dF} / \mathrm{F}$, in per cent with $0.1 \%$ ticks along the axis) are plotted against time (s). Mean membrane depolarization leads to decreased fluorescent intensity. Horizontal bar indicates the $2 \mathrm{~s}$ stimulus timing. Each curve gives the spatial average of a quadratic box comprising five adjacent pixels (much less than the size of a glomerulus), averaged over eight measurements within a period of 54 min. No further averaging or filtering is applied. The thin line is air control (change of syringe, but with no odour), thick line hexanol, dotted for citral, and dash-dot for hexanal. C and D show the responses at two different glomeruli: position $\mathrm{C}$ responds strongly to hexanol, but also to citral and hexanal, while position $\mathrm{D}$ is selective for hexanol, and gives no response to either hexanal or citral. Note that total bleaching is in the range of $0.2 \% \mathrm{dF} / \mathrm{F}$, and signals between 0.1 and $0.3 \% \mathrm{dF} / \mathrm{F}$. Different experiment from Fig. 3A, B.
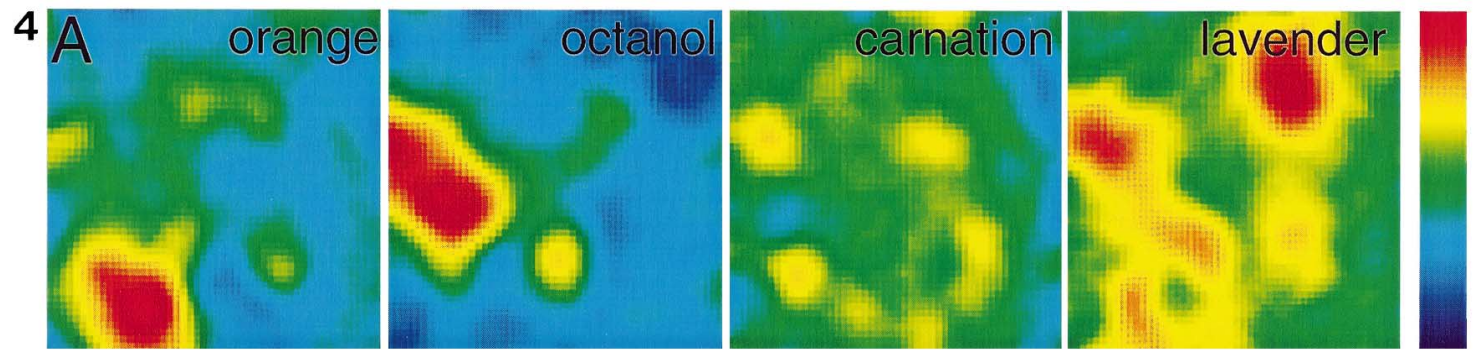

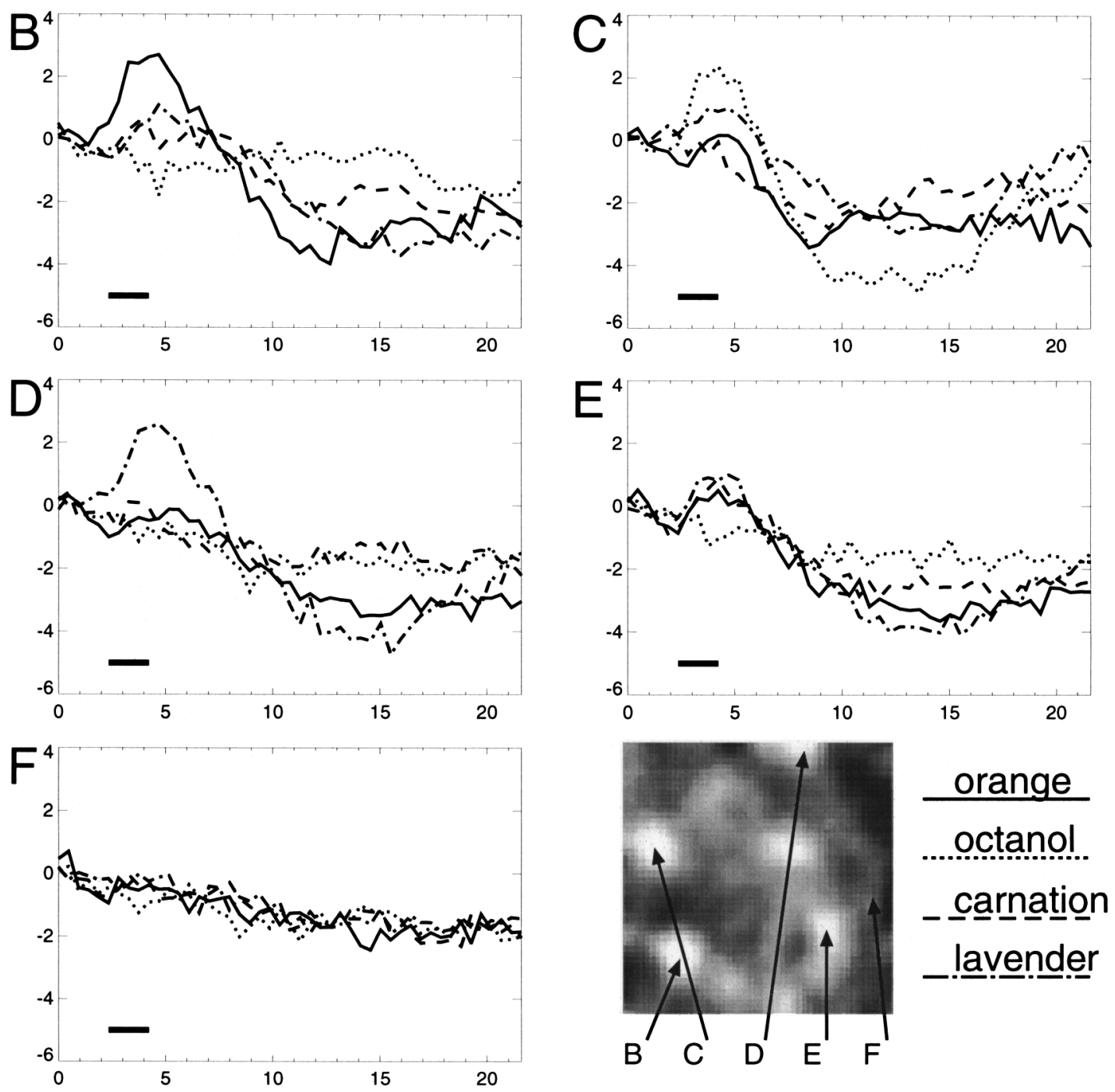

Fig. 4. Calcium signals in the antennal lobe (calcium-green). (A) False colour coded signals of antennal lobe measurements. The frames give the mean signal over the stimulus period, from left to right, as response to: orange, octanol, carnation and lavender. Side length is $250 \mu \mathrm{m}$, the view of the antennal lobe is comparable to Fig. 3A. The spatial resolution is $50 \times 50$ pixels, i.e. $5 \mu \mathrm{m}$ side length for each pixel. Images are spatially low-pass filtered with a $5 \times 5$ pyramidal filter. Colour-coding is equal for all frames, and given right to the figure. Maximum signals $(\mathrm{dF} / \mathrm{F}=2 \%)$ are coded red, minimum blue $(\mathrm{dF} / \mathrm{F}=-2 \%)$. (B-F) Time-courses of signals $(\% \mathrm{dF} / \mathrm{F}$ plotted against s). Calcium-green gives positive fluorescent intensity change for increased intracellular calcium concentrations. The plots show the time-courses at different positions of the antennal lobe. The average signal of a $5 \times 5$ pixel box is given $(25 \times 25 \mu \mathrm{m})$. Glomeruli vary in diameter between 30 and $50 \mu \mathrm{m}$. The positions taken are shown to the bottom right side of the figure: this black and white view of the antennal lobe corresponds to the activity map for carnation (as shown in A). The contrast has been enhanced in order to emphasize the positions of the glomeruli. Same measurement as in A. Note that position B gives a strong respose for orange (continuous line), with little changes for the other odours. Position $\mathrm{C}$ has a strong response for octanol (dotted line), a weak response to orange and lavender, and no response to carnation. Position D gives strong signals for lavender, but no signals to the other odours. Position E gives weak responses to all odours but octanol. Position F is not excited by any of the tested odours.

satisfactory $\mathrm{s} / \mathrm{n}$ ratio, while calcium sensitive dyes allowed for single trial measurements. False colour coded spatial maps of odour evoked activity are calculated subtracting the air-control from the signal in order to correct for bleaching. The pseudo colour code represents the average signal values during the stimulation period (2s).

\section{Results}

\subsection{Measuring membrane potentials in the antennal lobe}

RH795 gives bright fluorescent staining of the tissue. In the antennal lobe, single glomeruli are clearly identifiable in the fluorescent picture measured with the 
CCD camera (Fig. 3A). Signals upon odour stimulation are in the range of $0.2 \%$ (Fig. 3C and 3D).

The spatial pattern of the signals match the glomerular layout of the antennal lobe (Fig. 3B). In this case, stimulation with hexanal led to just one glomerulus being strongly activated. The spatial extent of the activated region-corresponding exactly to one glomerulus - indicates that glomeruli are indeed functional processing units, and not mere morphological structures. Other odours lead to a combination of several glomeruli being electrically active (compare with calcium signals, Fig. 4A).

Fig. 3C and 3D show the time-courses of signals in different locations of the antennal lobe. Upon odour stimulation, the mean membrane potential is rapidly depolarized to a plateau level. This level is maintained for the entire duration of the stimulus. With stimulus offset, baseline levels are readily regained. The measured depolarisation depends upon the odour used for stimulation. Thus, each odour leads to a unique mosaic of glomeruli activated with varying intensity.

Fig. 3C and 3D also show the response to the control air stimulation. In this case, the olfactometer also switches between two different syringes, the clean 'airbackground' syringe, which is used for the constant air current, and the clean 'air-control' syringe, through which the air-flow is directed during the 2-s stimulus interval in the control trial. No change in membrane potential could be detected, showing that there is no stimulus-correlated artefact independent of the odourant. In particular, switching between syringes does not lead to changes in the air flow pressure that would stimulate mechanoreceptors of the bee antennae.

\subsection{Changes in intracellular calcium in the antennal lobe}

Fig. 4 shows signals in the antennal lobe measured after staining the brain with calcium-green 1-AM. Signals upon odour stimulation vary between around $1 \%$ (in E) and almost 4\% (in D). Fig. 4A gives false-colour coded activity images of the antennal lobe as response to the following odours: orange, octanol, carnation and lavender. Each odour elicits a specific spatial-activity map, which is reproducible and constant during the entire experiment over a period of several hours (Joerges et al., 1997). Here, the left antennal lobe has been imaged, ventral is to the top, left is lateral and right is medial. While orange strongly activates a glomerulus to the latero-dorsal side of the antennal lobe (bottom-left in the imaged frame), octanol activates a group of two adjacent glomeruli, laterally (second frame), and also a central glomerulus, weakly. Carnation (third frame) activates a whole range of different glomeruli, with only weak signals in each of them. Lavender also activates several glomeruli weakly, but in addition strongly acti- vates a single glomerulus medio-ventrally. The timecourses for various positions in the AL are depicted in Fig. 4B-4F. Glomeruli show different selectivities for the tested odours: glomerulus B is strongly activated by orange (compare with the false-colour coded frames in Fig. 4A), and weakly by carnation and lavender. Glomerulus $\mathrm{C}$ is not activated by carnation, but by all other odours, and most strongly by octanol. Glomerulus $\mathrm{D}$ seems to be selective for lavender. Glomerulus E responds to all odours but octanol. Bleaching over the measurement time is around 2\% (see Fig. $4 \mathrm{~F}$ ).

\section{Discussion}

Olfactory perception is a complex task to be solved by the brain, the mechanisms of which are only partly understood. By optically imaging the olfactory system of the honeybee brain, we have shown that stimulation with odours leads to a mosaic of glomeruli being activated in the primary neuropil, and the antennal lobe. The spatio/ temporal mosaic of activity in the antennal lobe is constant within one individual and characteristic for any particular odour (Joerges et al., 1997; 1996; Galizia et al., 1996). Furthermore, it is possible to relate the activity pattern to the glomerular layout of the antennal lobe (Fig. 3B). It appears, that coding of odours is accomplished in a distributed manner ('sparse code') along the entire pathway from receptors to higher brain centres: we show here that any given odour is coded by a different mosaic of activated glomeruli in the antennal lobe of the (female) worker honeybee, but every particular glomerulus recorded so far is involved in coding several odours (see Fig. 4A). This finding is in contrast to the sexual pheromone system in male insects, where dedicated receptor neurones innervate dedicated glomeruli (Hansson et al., 1992; Hildebrand, 1995). Here, one would expect that only these glomeruli are activated when stimulated with the sex pheromone. In our experiments worker bees were stimulated with general odours, and the response properties of receptor cells involved are not devoted to pheromone signals (Shepherd, 1994; Akers and Getz, 1993).

It has been hypothesized that odours are coded by sequences of activated cell assemblies, which would form the internal representation of each odour. The temporal pattern of their spiking activity could form an integral part of the olfactory code (Laurent, 1996; Laurent et al., 1996, Wehr and Laurent, 1996). With the temporal resolution of our setup, this aspect of the olfactory code cannot be investigated.

This is not the first time that optical imaging has been applied to the olfactory system: Kauer and co-workers have shown the spread of activity in the olfactory bulb of salamander (Cinelli and Kauer, 1992; Cinelli et al., 1995), and Friedrich et al., (Friedrich and Korsching, 
1996; Friedrich et al., 1996) monitored afferent activity to the olfactory bulb in zebrafish. Olfactory imaging has also been used in the insect brain (Okada et al., 1996), including the bee brain (Lieke, 1993). This is the first time, however, that signals could be reliably related to the glomerular layout of the structure of the antennal lobe. We think that the combination of low-temperature staining of the isolated head, where the dye has access to the nervous system from all sides, and the mechanical stable condition in the constantly perfused recording chamber were of paramount importance in developing this system.

Optical imaging of neuropils is always struggling with at least two great problems: signal to noise ratio, and uniform staining. Using voltage sensitive dyes gives good staining, but signals are below $1 \% \mathrm{dF} / \mathrm{F}$. Therefore great precaution must be taken to minimize noise, including spurious noise resulting from the preparation, movement artifacts, and noise in the recording system. The preparation presented here is particularly well suited. By cutting all muscles leading to the feeding apparatus, and removing thorax and abdomen from the preparation, we were able to reduce movements to below detection levels. Also, the plexiglas chamber allows for a cover glass to be thoroughly fixed in order to avoid any surface fluctuation deriving from the ringer flow. Thus, $\mathrm{s} / \mathrm{n}$ ratio appears now to be limited by shot noise due to the statistical nature of photons.

On the other hand, using calcium sensitive dyes, signals are greater (up to $5 \% \mathrm{dF} / \mathrm{F}$ ), allowing for singletrial measurements. However, staining of tissues with these dyes appears to be a problem in many preparations. With our cold-temperature bath immersion staining chamber, we were able to reliably and homogeneously load the brain cells with ester-coupled dyes. Since all glands and tracheae, as well as the neurolemma, have been removed from the brain at this stage, the ester coupled dye can reach the neurones directly. We also find that with calcium green an increase in fluorescence upon stimulation, i.e. an increase in intracellular calcium concentration, is often followed by a negative signal (Fig. 4). This slow and negative component could be due to several mechanisms, like an overshoot of calcium pumps, a component of intrinsic signals, or internalization of calcium green in intracellular stores. In the latter case, the late negative signal could reflect a decrease in calcium concentration within the intracellular stores due to second messenger activation, while the early positive component would be due to calcium influx from the extracellular space. Investigations are under way to separate these two components of the signal. We don't think that intrinsic signals are a significant part of our signal because measurements without dyes and the same preparation do not give any signals. Furthermore, the slow, negative component observed in calcium measurements is missing when voltage sensitive dyes are used, while the early portion of the signal is positive with the dye calcium green, and negative with RH795. The correspondence of the activity maps measured with either voltage sensitive dyes or ion sensitive dyes has been shown in turtels (Falk et al., 1996). In our system, signal amplitudes are always much lower than for single cell measurements. Since the entire tissue is stained, also brain regions out of focus will contribute to the overall light intensity, increasing the total light level $(\mathrm{F})$, without increasing the absolute light changes $(\mathrm{dF})$ measured in focus. This leads to a reduction in $\mathrm{dF} / \mathrm{F}$. Averaging of several trials when using voltage dependent dyes would spoil the quality of the data if signals varied between trials. However, we believe them to be good estimates of the activity in the antennal lobe because we could show, using single trial measurements with calcium-sensitive dyes, that the elicited patterns are broadly constant over a period of several hours and after repeated presentations of the same odour. Slight variations are certainly due in part to measurement noise (shot noise, perturbations in the setup, etc.). However, we suspect that part of this variability may also be due to a still eluent biological component, possibly reflecting the dependence of odour coding on motivational status or changes in odour coding as a consequence of the repeated presentation of the same odours, which might habituate the neurons involved, or might lead to a change of attention or motivation, or to learning because the odours might have had a meaning to the bee from former exposures to the odour in the field. It is not yet clear whether learning-related processes lead to changes in the representation of odours in the antennal lobe. Furthermore, the situation is probably dependent on the imaged brain region. The mushroom bodies, for example, are known to be a centre of plasticity for odour associated appetitive learning (Hammer and Menzel, 1995; Menzel and Müller, 1996). Using voltage sensitive dyes and averaging over several trials in this structure would possibly mask some of these effects. Further studies are necessary to address such effects.

Preliminary results from imaging other brain regions show that this preparation can be used to measure neuronal activity in any superficial neuropil in the brain. For example, odour stimulation leads to odour specific activity patterns in the mushroom body, as has been shown with this preparation using calcium sensitive dyes (Joerges et al., 1996). This preparation and recording chamber allows for optical recording of the entire honeybee brain, enabling us to follow up the way the brain handles information across the different stages of this process. Though we only imaged the olfactory system so far, the preparation should be apt for analysis of other activities, like processing of optical information, or the formation of memories. Since none of the steps involved in the preparation are peculiar to the bee brain, we consider this preparation to be of great potentiality for all insect physiologists. 


\section{Acknowledgements}

Dedo Koppitz, for mechanical assistance. Uwe Greggers, for electronic expertise. Eavan McGee, for correcting the English.

\section{References}

Akers RP, Getz WM. Response of olfactory receptor neurons in honeybees to odourants and their binary mixtures. J Comp Physiol A 1993;173:169-85.

Blasdel GG. Differential imaging of ocular dominance and orientation selectivity in monkey striate cortex. J Neurosci 1992;12:3115-38.

Boeckh, J, Distler, P, Ernst, KD, Hösl, M, Malun, D. Olfactory bulb and antennal lobe. In: Schild D, editor, Chemosensory Information Processing, Berlin:Springer-Verlag, 1990;201-27.

Bonhoeffer T, Grinvald A. The layout of iso-orientation domains in area 18 of cat visual cortex: Optical imaging reveals a pinwheellike organization. J Neurosci 1993;13:4157-80.

Bonhoeffer T, Kim DS, Malonek D, Shoham D, Grinvald A. Optical imaging of the layout of functional domains in area 17 and across the area $17 / 18$ border in cat visual cortex. Eur J Neurosci 1995;7:1973-88.

Borst A, Egelhaaf M. Dendritic processing of synaptic information by sensory interneurons. Trends Neurosci 1994;17:257-63.

Brotz TM, Egelhaaf M, Borst A. A prepatation of the blowfly (Calliphora erythrocephala) brain for in vitro electrophysiological and pharmacological studies. J Neurosci Methods 1995;57:37-46.

Cinelli AR, Kauer JS. Voltage-sensitive dyes and functional-activity in the olfactory pathway. Annu Rev Neurosci 1992;15:321-51.

Cinelli AR, Hamilton KA, Kauer JS. Salamander olfactory bulb neuronal activity observed by video rate, voltage-sensitive dye imaging. III. Spatial and temporal properties of responses evoked by odourant stimulation. J Neurophysiol 1995;73:2053-71.

Cohen LB. Optical measurements of action potential activity in invertebrate ganglia. Annu Rev Physiol 1989;51:527-41.

Falk CX, Cohen LB, Senseman DM. Comparison of optical signals in the turtle cns generated by voltage- and ion-sensitive dyes. Soc Neurosci Abstr 1996;22:321.

Friedrich RW, Korsching SI. Representation of odourant information by spatial afferent activity patterns in the zebrafish olfactory bulb. Soc Neurosci Abstr 1996;22:1072.

Friedrich, RW, Baier, H, Korsching, SI. Representation of odourant information by activity patterns in the zebrafish olfactory bulb: a study using voltage-sensitive dyes for axon tracing and optical recording. In: Elsner, N Schnitzler, H-U editors, Göttingen Neurobiology Report 1996, Stuttgart, New York: G. Thieme, 1996, pp. 296.

Galizia, CG, Küttner, A, Joerges, J, Menzel, R. 1996. Olfactory coding in the honeybee's antennal lobes revealed by optical imaging of $\mathrm{Ca}^{2+}$ and voltage sensitive dyes, Neurowissenschaftliche Gesellschaft, (Abstract).
Grinvald A, Lieke EE, Frostig RD, Gilbert CD, Wiesel TN. Funcional architecture of cortex revealed by optical imaging of intrinsic signals. Nature 1986;324:361-4.

Hammer M, Menzel R. Learning and memory in the honeybee. J Neurosci 1995;15:1617-30.

Hansson BS, Ljungberg H, Hallberg E, Löfstedt C. Functional specialization of olfactory glomeruli in a moth. Science 1992;256:1313-5.

Hildebrand JG. Analysis of chemical signals by nervous systems. Proc Natl Acad Sci USA 1995;92:67-74.

Joerges J, Galizia CG, Menzel R. Population codes for odours and their mixtures in the antennal lobes and mushroom bodies of the honeybee revealed by in vivo calcium imaging. Soc Neurosci Abstr 1996;22:1075.

Joerges, J, Küttner, A, Menzel, R. Optical imaging of the honeybee olfactory system. In: Elsner N, Menzel, R. editors, Göttingen Neurobiology Report 1995, G. Thieme, Stuttgart, New York: G. Thieme, 1995:424.

Joerges J, Küttner A, Galizia CG, Menzel R. Representations of odours and odour mixtures visualized in the honeybee brain. Nature 1997;387:285-8.

Kleinfeld D, Delaney KR, Fee MS, Flores JA, Tank DW, Gelperin A. Dynamics of propagating waves in the olfactory network of a terrestrial mollusk: An electrical and optical study. J Neurophysiol 1994;72:1402-19.

Laurent G. Odour images and tunes. Neuron 1996;16:473-6.

Laurent G, Wehr M, Davidowitz H. Temporal representations of odours in an olfactory network. J Neurosci 1996;16:3837-47.

Lieke EE. Optical recording of neuronal activity in the insect central nervous system: odourant coding by the antennal lobes of honeybees. Eur J Neurosci 1993;5:49-55.

Lieke EE, Frostig RD, Arieli A, Ts'o DY, Hildesheim R, Grinvald A. Optical imaging of cortical activity. Annu Rev Physiol 1989;51:543-9.

Menzel R, Müller U. Learning and memory in honeybees: from behavior to neural substrates. Annu Rev Neurosci 1996;19:379404.

Okada K, Kanzaki R, Kawachi K. High-speed voltage-sensitive dye imaging of an in vivo insect brain. Neurosci Lett 1996;209:197200.

Shepherd GM. Principles of specificity and redundancy underlying the organization of the olfactory system. Microsc Res Tech 1993;24:106-12.

Shepherd GM. Discrimination of molecular signals by the olfactory receptor neuron. Neuron 1994;13:771-90.

Ts'o DY, Frostig RD, Lieke EE, Grinvald A. Functional organization of primate visual cortex revealed by high resolution optical imaging. Science 1990;249:417-20.

Wehr M, Laurent G. Odour encoding by temporal sequences of firing in oscillating neural assemblies. Nature 1996;384:162-6.

Yuste R, Katz LC. Control of postsynaptic $\mathrm{Ca}^{2+}$ influx in developing neocortex by excitatory and inhibitory neurotransmitters. Neuron 1991;6:333-44.

Yuste R, Nelson DA, Rubin WW, Katz LC. Neuronal domains in developing neocortex: Mechanisms of coactivation. Neuron 1995;14:7-17. 